\title{
Cálculo de integrais definidas utilizando distribuições de probabilidades
}

\author{
Calculation of definite integrals using probability distributions
}

\begin{abstract}
Fernanda Vital de Paula Universidade Federal do Norte do Tocantins (UFNT), Campus Araguaína, Araguaína, TO, Brasil iD https://orcid.org/0000-0002-7936-8937, fernandavital@uft.edu.br Kevellyn Samara Lima da Silva Universidade Federal do Norte do Tocantins (UFNT), Campus Araguaína, Araguaína, TO, Brasil https://orcid.org/0000-0003-4327-9649, kevellynsamaral@gmail.com Domingos Santana Nascimento dos Santos Colégio Estadual Juscelino Kubistheck de Oliveira (CEJKO), Luzinópolis, TO, Brasil https://orcid.org/0000-0001-8231-7906, domingossns@hotmail.com
\end{abstract}

\section{Informações do Artigo}

\section{Como citar este artigo}

PAULA, Fernanda Vital de; SILVA, Kevellyn Samara Lima da; SANTOS, Domingos Santana Nascimento dos. Cálculo de integrais definidas utilizando distribuições de probabilidades. REMAT: Revista Eletrônica da Matemática, Bento Gonçalves, RS, v. 7, n. 1, p. e3014, 31 maio 2021. DOI:https://doi.org/10. 35819/remat2021v7i1id4694

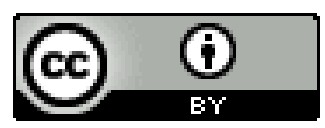

Histórico do Artigo

Submissão: 16 de outubro de 2020.

Aceite: 14 de abril de 2021 .

\section{Palavras-chave}

Distribuições de Probabilidades

Integral Definida

Cálculo

Probabilidade

\section{Resumo}

Este artigo é um recorte do trabalho de conclusão de curso de um dos autores e é direcionado aos interessados em conceitos das disciplinas de Cálculo Integral e Probabilidade. Neste sentido, uma técnica alternativa para resolução de integrais definidas e, até então, não apresentada na literatura, conforme as pesquisas realizadas, é proposta. Para o desenvolvimento deste artigo, as funções de distribuição de probabilidade das distribuições Beta e Exponencial, suas propriedades e o Teorema Fundamental do Cálculo serão enfatizados como suporte teórico ao leitor. Posteriormente, duas integrais definidas são resolvidas para exemplificação da técnica proposta, seguindo-se para a generalização das funções que podem ser integradas por meio das distribuições Exponencial e Beta. É possível evidenciar que o método proposto, além de consistir em um procedimento alternativo para a resolução de integrais definidas, oferece a vantagem de redução dos cálculos necessários quando utilizadas as técnicas de integração propostas nas literaturas de Cálculo Integral. 


\section{Keywords}

Probability Distributions

Definite Integrals

Calculation

Probability

\begin{abstract}
This article is a part of a monograph of one of the authors and is directed to those interested in concepts from the subjects of Integral Calculus and Probability. In this sense, it is proposed an alternative technique for solving definite integrals and until then not presented in the literature, according to the research carried out. For the development of this article, the probability distribution functions of the Beta and Exponential distributions, their properties and the Fundamental Theorem of Calculus will be emphasized as theoretical support to the reader. Subsequently, two definite integrals are solved to exemplify the proposed technique, followed by the generalization of the functions that can be integrated through the Exponential and Beta distributions. It is possible to show that the proposed method, in addition to being an alternative procedure for the resolution of definite integrals, offers the advantage of reducing the necessary calculations when using the integration techniques proposed in the Integral Calculus literature.
\end{abstract}

\section{Introdução}

Todo curso de Probabilidade exige, como pré-requisito, o conhecimento de técnicas utilizadas para a integração de funções. Esta condição se faz necessária para o cálculo de probabilidades no caso em que a variável de interesse é contínua. Para esse cálculo, a função densidade de probabilidade referente à variável é integrada no intervalo de interesse. Assim, nota-se uma linha tênue entre o processo de integração, em que diversas técnicas são utilizadas para a obtenção do resultado de integrais definidas, e o cálculo de probabilidades para variáveis aleatórias contínuas. Baseando-se nessa ideia, um questionamento possível é sobre a reciprocidade deste processo. Seria possível obter o resultado de integrais definidas por meio do conhecimento acerca do cálculo e distribuições de probabilidades?

É indiscutível a importância das integrais, sendo elas definidas ou indefinidas, pois possuem uma vasta aplicação em inúmeras áreas. Neste sentido, Stewart (2016) faz referência a aplicações da integração em Matemática no cálculo de áreas entre curvas, volumes, valor médio de uma função, comprimento de arcos, entre outros. O autor apresenta ainda aplicações à Física, Engenharia, Economia e Biologia, em exemplos que abordam pressão hidrostática e força, momentos e centros de massa, excedente do consumidor e capacidade cardíaca, respectivamente. Diante de inúmeras aplicações possíveis, diversos métodos foram desenvolvidos para resolução analítica de integrais ao longo do tempo, porém, a utilização dessas técnicas podem resultar em manipulações algébricas onerosas. Encontrar métodos analíticos que facilitem o cálculo de integrais definidas possui extrema importância em todas as áreas que utilizam o Cálculo Integral. 
Assim, este artigo tem o objetivo de apresentar um método alternativo para o cálculo de integrais definidas utilizando distribuições de probabilidade contínuas, tratando-se de um recorte da monografia de Santos (2014). Para compreensão deste método é preciso ter um conhecimento sobre variáveis aleatórias contínuas e técnicas de integração. Neste sentido, para o desenvolvimento do artigo, alguns conceitos e definições sobre variáveis aleatórias contínuas serão abordados brevemente, dando ênfase às funções de distribuições de probabilidades e esperança matemática dos modelos Beta e Exponencial. Além disso, algumas definições e propriedades sobre integrais definidas serão apresentadas tais como o Teorema Fundamental do Cálculo e algumas técnicas usuais de integração. Para um maior aprofundamento teórico do leitor no que se refere a estes itens, indica-se Casella e Berger (2011), Morettin (2010), Stewart (2016) e Thomas (2012).

\section{Preliminares}

Uma variável aleatória (VA) é uma função que associa a cada um dos eventos de um espaço amostral um único número real. Ela pode ser classificada em discreta ou contínua. Neste estudo, apenas o conhecimento sobre a VA contínua (VAC) se faz necessário, dada sua estreita relação com processos de integração no cálculo de probabilidades. Uma VAC pode assumir qualquer valor em um intervalo e é obtida, geralmente, por meio de medições de tempo, peso, altura e outros.

Associada à VAC, tem-se a função de densidade de probabilidade (FDP), determinada aqui como $f$, que deve satisfazer as seguintes condições:

$\left.1^{a}\right) f(x) \geqslant 0$,

$\left.2^{\mathrm{a}}\right) \int_{a}^{b} f(x) d x=1$,

em que $f$ é definida no intervalo $[a, b]$. Sobre os limites do intervalo, $a$ e $b$ podem assumir $-\infty$ ou $+\infty$, respectivamente.

Algumas variáveis aleatórias seguem certos padrões e podem ser modeladas por distribuições de probabilidades conhecidas. Dadas as propriedades de tais distribuições, a resolução de problemas envolvendo a VA em questão pode ser facilitada. Como exemplificação de distribuições conhecidas relacionadas às VACs, a Exponencial e Beta podem ser citadas.

No que se refere à distribuição Beta, ela é frequentemente usada para modelagem de variáveis que assumem valores no intervalo $(0,1)$, caracterizando-se como uma boa opção para modelar 
proporções. Se $X$ se distribui conforme uma Beta com parâmetros $\alpha$ e $\beta$, isto é $X \sim \operatorname{Beta}(\alpha, \beta)$, então sua FDP é ser dada por:

$$
f(x)=\frac{1}{B(\alpha, \beta)} x^{\alpha-1}(1-x)^{\beta-1}, \quad x \in(0,1), \quad \alpha>0, \quad \beta>0 .
$$

A função Beta, indicada pelo fator $B(\alpha, \beta)$, está associada à função Gama, da seguinte forma:

$$
B(\alpha, \beta)=\int_{0}^{1} x^{\alpha-1}(1-x)^{\beta-1} d x=\frac{\Gamma(\alpha) \Gamma(\beta)}{\Gamma(\alpha+\beta)} .
$$

Dada essa relação, propriedades da função Gama são essenciais ao lidar com a função Beta. Algumas das principais são citadas a seguir.
i. $\Gamma(1)=1$;
ii. $\Gamma(n+1)=n \Gamma(n)$;
iii. $\Gamma(n)=(n-1)$ !.

A distribuição Exponencial, considerada a mais simples em termos matemáticos, é caracterizada por ter uma função de taxa de falha constante. Normalmente é usada para modelar variáveis como o tempo de vida. Caso uma VAC $X$ se distribua exponencialmente com parâmetro $\beta$, isto é, $X \sim \operatorname{Exp}(\beta)$, sua FDP será dada por:

$$
f(x)=\frac{1}{\beta} e^{-\frac{x}{\beta}}, \quad \beta>0, \quad x \geq 0 .
$$

Quando apresentado um problema, por exemplo, em que $X$ é uma VAC que descreve as horas de funcionamento de uma lâmpada e tem-se o interesse de calcular a probabilidade de uma lâmpada parar de funcionar após 800 horas de uso, o modelo Exponencial com certo parâmetro $\beta$ é adequado. Nesse caso, a FDP da Exponencial deve ser integrada no intervalo [0,800], ou seja, a integral, $\int_{0}^{800} \frac{1}{\beta} e^{-\frac{x}{\beta}} d x$ deve ser resolvida. Para isso, a técnica de substituição do Cálculo Integral deve ser utilizada. Assim, observa-se a estreita ligação entre o cálculo de probabilidades para VAC e o cálculo de integrais definidas.

Do último, emerge a indispensabilidade da consideração do Teorema Fundamental do Cálculo (TFC), nesse artigo. Destaca-se que o TFC é um dos conceitos mais importantes do Cálculo Integral que se fundamentou a partir do problema relacionado ao cálculo de áreas.

Thomas (2012) apresenta o TFC em duas partes. A primeira mostra que se uma função $f(t)$ é integrável no intervalo finito $I$, a integral de qualquer número $a \in I$ até um número $x \in I$ determina 
uma nova função, $F(x)=\int_{a}^{x} f(t) d t$ com limite superior e inferior em $a$ e $x$, respectivamente. Sendo $f$ uma função contínua, a derivada desta nova função, $F(x)$, será igual à $f(x)$. Isto é, existe uma função $F(x)$ que é a primitiva de $f(x)$. Sobre a segunda parte do teorema, pode-se dizer que calcular a integral de $f(x)$, sendo $f$ contínua no intervalo $[a, b]$, é calcular a diferença de $b$ aplicado na primitiva de $f(x)$ por $a$ aplicado na primitiva de $f(x)$, ou seja, $F(b)-F(a)$.

Para o cálculo de integrais definidas, mesmo dispondo do TFC, a necessidade de resolução das integrais não é poupada. A resolução de algumas integrais exige um nível de dificuldade elevado para a qual podem ser utilizadas algumas técnicas de integração. As técnicas de substituição e integração por partes podem ser citadas como exemplos. No primeiro caso, a ideia é substituir uma função relativamente complicada por uma mais simples trocando $x$ por $u$,

$$
\int f(g(x)) g^{\prime}(x) d x=\int f(u) d u \text {. }
$$

Já a integração por partes deriva da regra do produto do cálculo diferencial tendo o seguinte formato:

$$
\int f(x) g^{\prime}(x) d x=f(x) g(x)-\int g(x) f^{\prime}(x) d x
$$

Para uma maior praticidade faz-se $u=f(x)$ e $d v=g(x)$. Feita esta substituição deriva-se $u$ e integra-se $v$,

$$
f(x) g(x)-\int f(x) g^{\prime}(x) d x=u v-\int v d u
$$

O que se espera com esse processo é que a integral obtida tenha uma resolução mais simples do que a inicial.

Ainda sobre variáveis aleatórias, uma medida de posição de relativa importância para a compreensão do comportamento de uma VA é a esperança matemática.

A esperança matemática é o que se espera que ocorra na realização de uma VA, podendo ser interpretada como o centro da distribuição de probabilidade, de acordo com Morettin (2010). No caso da VAC $X$ com FDP $f$ definida em $[a, b]$, ela é calculada da seguinte forma:

$$
\mathbb{E}(X)=\int_{a}^{b} x f(x) d x
$$

Como exemplificação, se $X \sim \operatorname{Beta}(\alpha, \beta)$, com sua FDP representada por $f$, tem-se:

$$
\mathbb{E}(X)=\int_{0}^{1} x f(x) d x=\frac{\alpha}{\alpha+\beta} .
$$


No caso em que $X \sim \operatorname{Exp}(\beta)$, com sua FDP representada por $g$, tem-se:

$$
\mathbb{E}(X)=\int_{0}^{+\infty} x f(x) d x=\frac{1}{\beta}
$$

\section{Resultados principais}

Nesta seção serão apresentadas as resoluções de duas integrais definidas pela utilização de distribuições de probabilidades contínuas. Anterior à apresentação das resoluções, estas serão resolvidas por técnicas usuais do Cálculo Integral apresentadas na seção anterior.

Para tal, na resolução primeira integral utiliza-se a distribuição Beta e, no que se refere à segunda integral, lança-se mão da distribuição Exponencial.

\subsection{Distribuição Beta}

O cálculo da integral da função $f(x)=(1-x)^{9}$, que denominaremos $F(x)$, é normalmente efetuado utilizando a técnica da substituição:

$$
u=1-x \Rightarrow d u=-d x \quad \text { ou } \quad-d u=d x .
$$

Assim,

$$
\begin{aligned}
& \int(1-x)^{9} d x \\
= & \int u^{9}(-d u) \\
= & -\int u^{9} d u \\
= & -\frac{u^{10}}{10}+C .
\end{aligned}
$$

Retornando a variável $u$ para $x$, obtém-se:

$$
F(x)=-\frac{(x-1)^{10}}{10}+C
$$


Pelo TFC, segue que:

$$
\begin{aligned}
& \int_{0}^{1}(1-x)^{9} d x \\
= & \left.F(x)\right|_{0} ^{1} \\
= & F(1)-F(0) \\
= & -\frac{0^{10}}{10}+C-\left[-\frac{(-1)^{10}}{10}+C\right] \\
= & \frac{1}{10} .
\end{aligned}
$$

Portanto,

$$
\int_{0}^{1}(1-x)^{9} d x=\frac{1}{10}
$$

Ao realizar algumas manipulações algébricas, é possível resolver essa integral de uma forma alternativa utilizando a distribuição Beta. Para isso, faz-se necessário manipular a função que precisa ser integrada de maneira que ela assuma a forma da FDP da distribuição Beta.

Tendo como base que a FDP da distribuição Beta é dada por $\frac{1}{B(\alpha, \beta)} x^{\alpha-1}(1-x)^{\beta-1}$ e como a função a ser integrada é $f(x)=(1-x)^{9}$, tem-se:

$$
\begin{aligned}
& (1-x)^{9} \\
= & x^{1-1}(1-x)^{10-1} \\
= & x^{1-1}(1-x)^{10-1} \frac{B(1,10)}{B(1,10)} \\
= & B(1,10) \frac{x^{1-1}(1-x)^{10-1}}{B(1,10)} .
\end{aligned}
$$

Portanto,

$$
f(x)=B(1,10) g(x),
$$

em que

$$
g(x)=\frac{x^{1-1}(1-x)^{10-1}}{B(1,10)} .
$$


Logo, $g(x)$ corresponde à FDP de uma $\operatorname{Beta}(1,10)$, sendo $\int_{0}^{1} g(x)=1$. Tal igualdade ocorre em consequência da $2^{-a}$ condição de uma FDP. Desta forma,

$$
\begin{aligned}
& \int_{0}^{1}(1-x)^{9} d x \\
= & \int_{0}^{1} B(1,10) g(x) d x \\
= & B(1,10) \int_{0}^{1} g(x) d x \\
= & B(1,10) \cdot 1 \\
= & B(1,10) \\
= & \frac{\Gamma(1) \Gamma(10)}{\Gamma(10+1)} \\
= & \frac{1 \cdot \Gamma(9+1)}{\Gamma(10+1)} \\
= & \frac{1 \cdot 9 !}{10 \cdot 9 !} \\
= & \frac{1}{10} .
\end{aligned}
$$

Outras integrais podem ser resolvidas através deste método desde que seja possível manipular a função a ser integrada de tal modo que ela assuma a forma de uma FDP de uma distribuição conhecida.

\subsection{Distribuição Exponencial}

No que se refere à resolução da integral

$$
\int_{0}^{+\infty} x e^{-x} d x
$$

a técnica de integração comumente usada é a integração por partes:

$$
\begin{aligned}
& u=x \quad \text { e } \quad d v=e^{-x} \\
& d u=d x \quad \text { e } \quad v=-e^{-x}
\end{aligned}
$$

Assim,

$$
\begin{aligned}
F(x) & =\int x e^{-x} d x \\
& =-x e^{-x}-\int-e^{-x} d x \\
& =-x e^{-x}-e^{-x} \\
& =-e^{-x}(x+1) .
\end{aligned}
$$


Deste modo, pelo TFC, segue que

$$
\begin{aligned}
& \int_{0}^{+\infty} x e^{-x} d x \\
= & \lim _{a \rightarrow+\infty}\left[\int_{0}^{a} x e^{-x} d x\right] \\
= & \lim _{a \rightarrow+\infty}[F(a)-F(0)] \\
= & \lim _{a \rightarrow+\infty}\left[-e^{-a}(a+1)-\left[-e^{0}(0+1)\right]\right. \\
= & \lim _{a \rightarrow+\infty}\left[-\frac{(a+1)}{e^{a}}+1\right] \\
\triangleq & \lim _{a \rightarrow+\infty}\left[-\frac{1}{e^{a}}+1\right] \\
= & 1 .
\end{aligned}
$$

A igualdade indicada por $\triangle$ justifica-se pela Regra de L'Hôspital, que pode ser consultada em Stewart (2016).

No caso da metodologia de resolução de integrais definidas propostas neste trabalho, será considerada a FDP da distribuição exponencial que é dada por:

$$
f(x)=\frac{1}{\beta} e^{-\frac{x}{\beta}}, \quad \beta>0, \quad 0 \leq x \leq \infty .
$$

É possível reescrever $h(x)=x e^{-x}$ para que a integral apresentada seja resolvida por meio da distribuição exponencial, da seguinte maneira:

$$
h(x)=x \frac{1}{1} e^{-\frac{x}{1}}=x g(x),
$$

em que $g(x)$ equivale à FDP de $\operatorname{Exp}(1)$. Deste modo, como já apresentado a esperança da distribuição Exponencial, tem-se:

$$
\int_{0}^{+\infty} x e^{-x} d x=\int_{0}^{+\infty} x g(x) d x=\mathbb{E}(X)=1,
$$

visto que $X \sim \operatorname{Exp}(1)$ e, portanto, $\mathbb{E}(X)=1$. Aqui, é possível perceber o quão podem ser reduzidos os cálculos para a obtenção do resultado de uma integral definida, quando as distribuições probabilísticas são utilizadas, conforme o método proposto.

Diante das duas resoluções de integrais definidas utilizando distribuições de probabilidade de VAC, observa-se que a relação mantida entre o cálculo de integrais definidas e a distribuição de probabilidade pode ser utilizada, em vez de apenas para calcular probabilidades em problemas envolvendo distribuições conhecidas em que o uso de integrais faz-se necessário, como exemplificado para as horas de funcionamento de uma lâmpada. Observa-se a reciprocidade nesse processo, 
podendo calcular integrais definidas por meio do conhecimento de distribuições de probabilidade de variáveis aleatórias contínuas, desde que seja possível manipular a função a ser integrada para que esta assuma a forma da FDP de uma distribuição conhecida.

Apesar de os exemplos de aplicação da metodologia apresentada terem sido pontuais, é possível generalizar a forma das funções que podem ser manipuladas a fim de que assumam a FDP das distribuições Beta ou Exponencial no processo de cálculo de integrais definidas.

\subsection{Generalização das funções integradas por meio das distribuições Beta e Exponencial}

A Tabela 1 apresenta a generalização das funções que podem ser integradas conforme as distribuições utilizadas neste artigo.

\begin{tabular}{|c|c|}
\hline Função & Distribuição \\
\hline $\begin{array}{r}f(x)=a x^{n}(b-b x)^{m}, \quad x \in(0,1) \quad n, m \geqslant 0, \quad a, b \in \mathbb{R} \\
g(x)=a x e^{-b x}, \quad a \in \mathbb{R}, \quad b>0, \quad x \geq 0\end{array}$ & $\begin{array}{c}\text { Beta } \\
\text { Exponencial }\end{array}$ \\
\hline
\end{tabular}

Fonte: Adaptado de Santos (2014, p. 64).

Os resultados exibidos são demonstrados a seguir.

\subsubsection{Distribuição Beta}

$$
\begin{gathered}
\int a x^{n}(b-b x)^{m} d x=a b^{m} \int x^{(n+1)-1}(1-x)^{(m+1)-1} d x=a b^{m} B(n+1, m+1) \int \frac{x^{(n+1)-1}(1-x)^{(m+1)-1}}{B(n+1, m+1)} d x \\
=a b^{m} B(n+1, m+1) \int f(x) d x,
\end{gathered}
$$

em que $f$ corresponde à FDP da distribuição Beta com parâmetros $n+1$ e $m+1$. No caso em que $[0,1]$ é o intervalo de variação de $x$, a integral definida de $a x^{n}(b-b x)^{m}$ resultará em $a b^{m} B(n+$ $1, m+1)$, tendo em vista que $\int_{0}^{1} f(x) d x=1$, conforme a $2^{-}$condição exibida na Seção 2 . Tal caso foi evidenciado no exemplo apresentado na Subseção 3.1 .

Assim, toda função do tipo $a x^{n}(b-b x)^{m}$ pode ser manipulada algebricamente de modo que recaia na FDP da distribuição Beta.

\subsubsection{Distribuição Exponencial}

$$
\int a x e^{-b x} d x=a \int x e^{-\frac{x}{b}} d x=a \cdot \frac{1}{b} \int \frac{1}{\frac{1}{b}} x e^{-\frac{x}{\frac{1}{b}}} d x=\frac{a}{b} \int x \frac{1}{\frac{1}{b}} e^{-\frac{x}{b}} d x=\frac{a}{b} \int x g(x) d x
$$


em que $g$ corresponde à FDP da distribuição Exponencial com parâmetro $\frac{1}{b}$. Nesse caso, a integral resultará na esperança da distribuição Exponencial. No caso em que $[0,+\infty)$ é o intervalo de variação de $x$, a integral definida de $a x e^{-b x}$ resultará em $a$, tendo em vista que $\int_{0}^{+\infty} x g(x) d x=$ $\mathbb{E}(X)=b$, conforme a esperança da distribuição Exponencial exibida na Seção 2. Tal caso foi evidenciado no exemplo apresentado na Subseção 3.2.

Em ambos os casos, para o cálculo de integrais definidas, faz-se necessário verificar se os parâmetros e o intervalo de variação da variável estão de acordo com as referidas distribuições. Mais detalhes acerca dos intervalos são dados a seguir.

\subsubsection{Sobre os intervalos de integração}

No caso em que o intervalo de integração corresponde ao intervalo de variação da variável que se distribui conforme o modelo considerado, como nos exemplos apresentados, o resultado da integral é obtido diretamente como foi possível observar, por meio de condições da FDP ou de propriedades como a esperança matemática.

Quando o intervalo de integração está contido no intervalo de variação da variável, de modo que ambos são distintos, é possível calcular a integral definida por meio da função de distribuição acumulada da variável, utilizando softwares específicos. Utilizando o $R$ - project como recurso, por exemplo, e revisitando o Exemplo da Subseção 3.1, tem-se:

$$
\int_{0,25}^{0,75}(1-x)^{9}=\int_{0,25}^{0,75} B(1,10) g(x) d x=B(1,10) \int_{0,25}^{0,75} g(x) d x=\frac{1}{10} \int_{0,25}^{0,75} g(x) d x,
$$

em que $g$ corresponde à FDP de uma $\operatorname{Bet} a(1,10)$. Desta forma,

$\int_{0,25}^{0,75}(1-x)^{9}=\frac{1}{10}[G(0,75)-G(0,25)]=\frac{1}{10}[\operatorname{pbeta}(0.75,1,10)-\operatorname{pbeta}(0.25,1,10)] \approx \frac{1}{10} .0,06 \approx 0,006$, em que pbeta $(0,75,1,10)$, por exemplo, é o código a ser executado no $R$ - project a fim de obter o valor da distribuição acumulada de $\operatorname{Beta}(1,10)$ em 0,75 , isto é, pbeta $(0,75,1,10)=G(0,75)=$ $\int_{0}^{0,75} g(x) d x$.

Nesse caso, não é possível calcular a integral sem a utilização de softwares, planilhas ou tabelas que fornecem valores da função de distribuição acumulada de uma variável.

A última situação a ser considerada, quando o intervalo de integração não está contido no intervalo de variação da variável, não foi explorada na pesquisa que originou este artigo. No entanto, suspeita-se que por meio de uma mudança de variável seja possível adequar os intervalos ao inter- 
valo de variação da variável como nos casos anteriores. Tal situação se constitui como um tópico de investigação em continuidade a essa pesquisa e não será exemplificada nesse artigo.

\section{Considerações finais}

O método alternativo apresentado consiste na resolução de integrais definidas utilizando o conhecimento acerca das distribuições de probabilidades contínuas. Para utilizá-lo, faz-se necessário o conhecimento e domínio dos conceitos e propriedades referentes aos modelos probabilísticos no que diz respeito ao conhecimento de seus parâmetros, intervalos de variação dos mesmos e esperança matemática. Neste sentido, sua utilização pode tornar o conhecimento referente às distribuições de probabilidade mais consistente, além de permitir a obtenção de resultados por meio de resoluções mais simples às oferecidas pelas técnicas usuais, como é observado no exemplo da Subseção 3.2 .

Para aplicação do método apresentado é importante destacar que é imprescindível verificar se o intervalo de integração está contido no intervalo de variação da variável à qual se refere a distribuição de probabilidade que será utilizada. Como exemplo, em $\int_{2}^{5} x^{2}(1-x)^{9} d x$, a função a ser integrada sugere o uso da distribuição Beta, como pode ser observado na Tabela 11, porém, $[2,5]$ não está contido em $[0,1]$, que é o intervalo de variação de uma variável que tem distribuição Beta. Nesse caso, a integral não pode ser resolvida por meio da distribuição Beta, de modo direto, como citado anteriormente. Nos casos em que a adequação dos intervalos é verificada, como observa-se em $\int_{\frac{1}{4}}^{\frac{5}{8}} x^{2}(1-x)^{9} d x$, basta realizar manipulações algébricas na função a ser integrada para que ela se apresente como a função densidade de probabilidade da distribuição Beta e utilizar softwares, planilhas ou tabelas que forneçam valores da função de distribuição acumulada da distribuição Beta.

Por fim, espera-se que este artigo possa contribuir com o ensino e aprendizado de conteúdos de Probabilidade e Cálculo Integral, que ele permita a relação entre as duas disciplinas e que o método possa se apresentar como uma boa alternativa quando as técnicas usuais resultarem em cálculos onerosos. 


\section{Referências}

CASELLA, G.; BERGER, R. L. Inferência estatística. 2. ed. São Paulo: Cengage Learning, 2011. MORETTIN, L. G. Estatística Básica. São Paulo: Pearson Prentice Hall, 2010.

SANTOS, D. S. N. Cálculo de integrais definidas utilizando funções de distribuição de probabilidade. Orientadora: Fernanda Vital de Paula. 2014. 35f. Monografia (Graduação em Matemática) - Universidade Federal do Tocantins, Araguaína, 2014.

STEWART, J. Cálculo. v. 1. Trad. EZ2 Translate. 7 ed. São Paulo: Cengage Learning, 2016.

THOMAS, G. B. Cálculo. v. 1. Trad. Kleber Pedroso e Regina Simille de Macedo. Revisão técnica Claudio Hirofume Asano. 12. ed. São Paulo: Pearson Education do Brasil, 2012. 